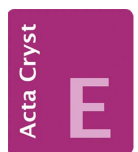

CRYSTALLOGRAPHIC COMMUNICATIONS

ISSN 2056-9890

Received 29 April 2020

Accepted 30 April 2020

Edited by G. Diaz de Delgado, Universidad de Los Andes, Venezuela

Keywords: crystal structure; piperidine; hydrogen bonding; Hirshfeld surface analysis; energy frameworks.

CCDC reference: 2000223

Supporting information: this article has supporting information at journals.iucr.org/e

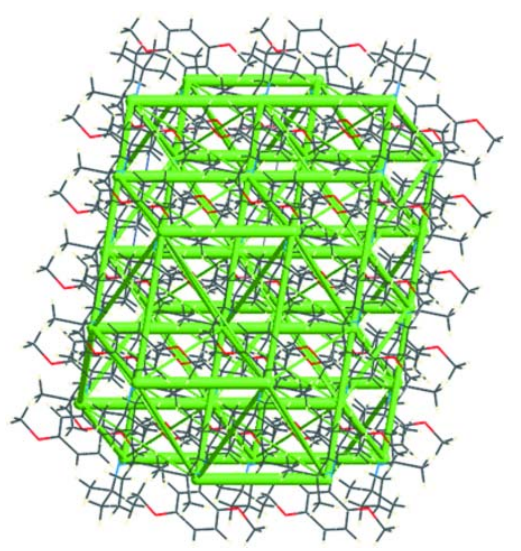

\section{The crystal structure and Hirshfeld surface analysis of 1-(2,5-dimethoxyphenyl)-2,2,6,6-tetramethyl- piperidine}

\author{
Mustapha Tiouabi, ${ }^{a}$ Raphaël Tabacchi ${ }^{\mathrm{a}}$ and Helen Stoeckli-Evans ${ }^{\mathrm{b} *}$
}

${ }^{\mathbf{a}}$ Institute of Chemistry, University of Neuchâtel, Av. de Bellevax 51, CH-2000 Neuchâtel, Switzerland, and ${ }^{\mathbf{b}}$ Institute of Physics, University of Neuchâtel, rue Emile-Argand 11, CH-2000 Neuchâtel, Switzerland. *Correspondence e-mail: helen.stoeckli-evans@unine.ch

In the title compound, $\mathrm{C}_{17} \mathrm{H}_{27} \mathrm{NO}_{2}$, the piperidine ring has a chair conformation and is positioned normal to the benzene ring. In the crystal, molecules are linked by $\mathrm{C}-\mathrm{H} \cdots \mathrm{O}$ hydrogen bonds, forming chains propagating along the $c$-axis direction.

\section{Chemical context}

During research on phytotoxins produced by the Ceratocystis fimbriata species (Tiouabi, 2005), the pathogenic agents responsible for the infections of plane, coffee and elm trees, analytical and spectroscopic studies enabled the isolation of a number of isocoumarins in small quantities. In order to confirm their molecular structures and especially to study their phytotoxicity and pathogenicity it was necessary to develop efficient methods for the total syntheses of these various isocoumarins. The title compound (3) was synthesized as a side product during the synthesis of the intermediate, methyl 3,6dimethoxy-2-(2-methoxy-2-oxoethyl)benzoate (2) (see Fig. 1), necessary for the total synthesis of the isocoumarin 5,8dimethoxy-3-methyl-1H-isochromen-1-one (Tiouabi, 2005).

\section{Structural commentary}

The molecular structure of the title compound, 1-(2,5-dimethoxyphenyl)-2,2,6,6-tetramethylpiperidine (3), is illustrated in Fig. 2. The piperidine ring has a chair conformation with atoms $\mathrm{N} 1$ and $\mathrm{C} 11$ being displaced by -0.5171 (12) and 0.6876 (15) $\AA$, respectively, from the mean plane of the

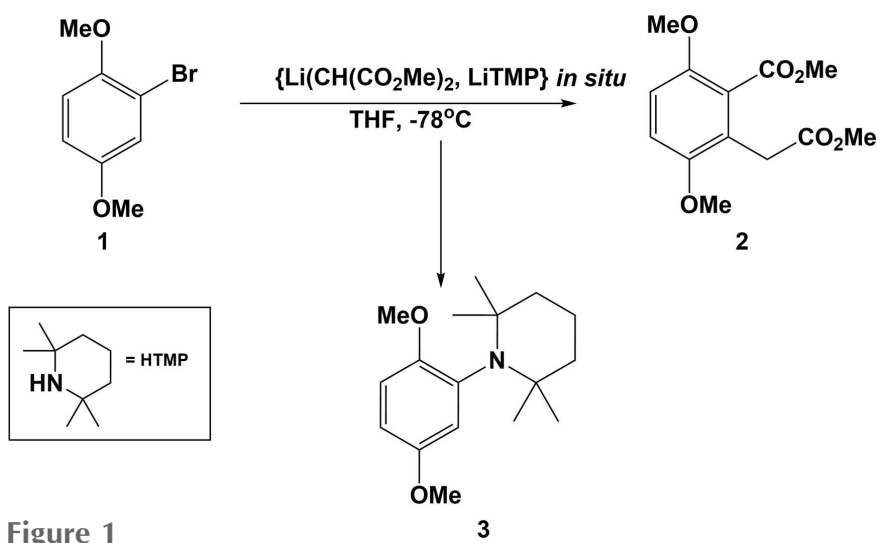

The reaction scheme resulting in the formation of the title compound, $\mathbf{3}$. 
remaining four $\mathrm{C}$ atoms $(\mathrm{C} 9 / \mathrm{C} 10 / \mathrm{C} 12 / \mathrm{C} 13)$. This mean plane is normal to the plane of the benzene ring (C1-C6), with a dihedral angle of $88.34(9)^{\circ}$. Planes $\mathrm{C} 2 / \mathrm{O} 1 / \mathrm{C} 7$ and $\mathrm{C} 5 / \mathrm{O} 2 / \mathrm{C} 8$, involving the methoxy groups, are inclined to the benzene ring by $13.23(15)$ and $10.45(15)^{\circ}$, respectively.

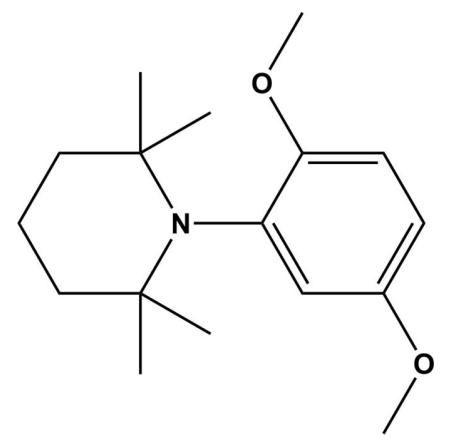

\section{Supramolecular features}

In the crystal of $\mathbf{3}$, molecules related by the glide plane are linked by $\mathrm{C}-\mathrm{H}$. . O hydrogen bonds, forming chains propagating along the $c$-axis direction (Fig. 3 and Table 1). There are no other significant intermolecular interactions present in the crystal.

\section{Hirshfeld surface analysis and two-dimensional fingerprint plots}

The Hirshfeld surface analysis (Spackman \& Jayatilaka, 2009) and the associated two-dimensional fingerprint plots (McKinnon et al., 2007) were performed with CrystalExplorer17.5 (Turner et al., 2017). For an excellent explanation of the use of Hirshfeld surface analysis and other calculations,

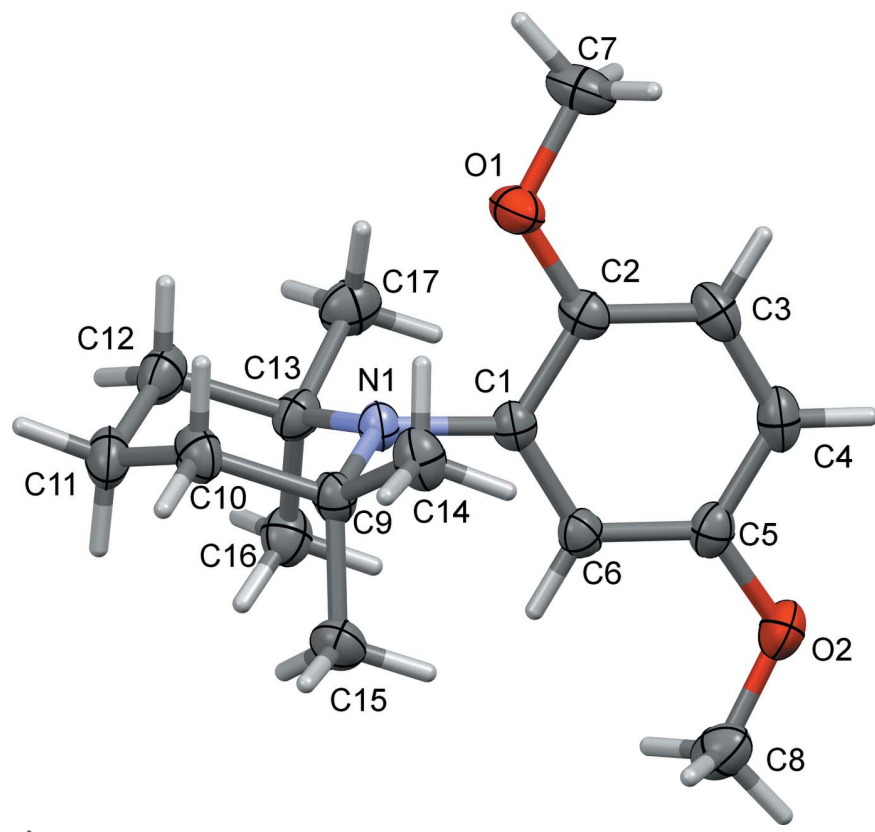

Figure 2

The molecular structure of compound $\mathbf{3}$, with atom labelling. Displacement ellipsoids are drawn at the $50 \%$ probability level.
Table 1

Hydrogen-bond geometry $\left(\AA,^{\circ}\right)$.

\begin{tabular}{lllll}
\hline$D-\mathrm{H} \cdots A$ & $D-\mathrm{H}$ & $\mathrm{H} \cdots A$ & $D \cdots A$ & $D-\mathrm{H} \cdots A$ \\
\hline $\mathrm{C} 8-\mathrm{H} 8 B \cdots \mathrm{O} 2^{\mathrm{i}}$ & 0.98 & 2.51 & $3.495(2)$ & 180 \\
\hline
\end{tabular}

Symmetry code: (i) $x,-y+\frac{1}{2}, z+\frac{1}{2}$.

Table 2

Short interatomic contacts $(\AA)^{a}$ in the crystal of compound $\mathbf{3}$.

\begin{tabular}{llr}
\hline Atom $1 \cdots$ Atom 2 & Length & Length $-\mathrm{vdW}$ \\
\hline $\mathrm{O} 2 \cdots \mathrm{H} 8 B^{\text {ii }}$ & 2.515 & -0.205 \\
$\mathrm{C} 4 \cdots \mathrm{H} 15 C^{\text {iii }}$ & 2.830 & -0.070 \\
$\mathrm{O} 2 \cdots \mathrm{H} 15 B^{\text {ii }}$ & 2.686 & -0.034 \\
$\mathrm{C} 2 \cdots \mathrm{H} 8 A^{\text {iv }}$ & 2.918 & 0.018 \\
$\mathrm{C} 3 \cdots \mathrm{H} 14 C^{\text {iii }}$ & 2.977 & 0.077 \\
$\mathrm{H} 7 C \cdot \mathrm{H} 17 A^{\text {iv }}$ & 2.484 & 0.084 \\
$\mathrm{O} 1 \cdots \mathrm{H} 16 A^{\text {iv }}$ & 2.814 & 0.094 \\
\hline
\end{tabular}

Note: (a) Calculated using Mercury (Macrae et al., 2020). Symmetry codes: (ii) $x,-y+\frac{1}{2}$, $z-\frac{1}{2}$; (iii) $x, y, z-1$; (iv) $x-1, y, z$.

such as energy frameworks, to study the molecular packing see the recent article by Tiekink and collaborators (Tan et al., 2019). The Hirshfeld surface is colour-mapped with the normalized contact distance, $d_{\text {norm }}$, from red (distances shorter than the sum of the van der Waals radii) through white to blue (distances longer than the sum of the van der Waals radii). The energy frameworks (Turner et al., 2015; Tan et al., 2019) are represented by cylinders joining the centroids of molecular pairs using red, green and blue colour codes for the $E_{\text {electrostatic }}$,

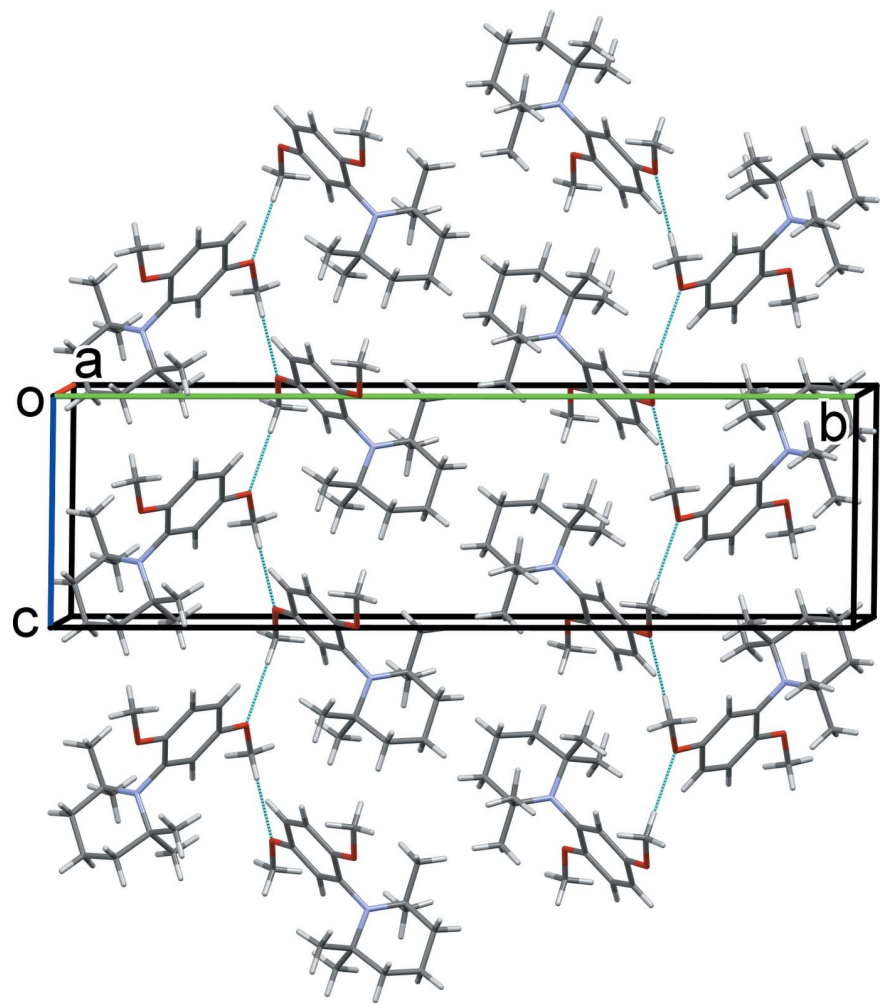

Figure 3

A view along the $a$ axis of the crystal packing of compound 3. The hydrogen bonds (Table 1) are shown as dashed lines. 


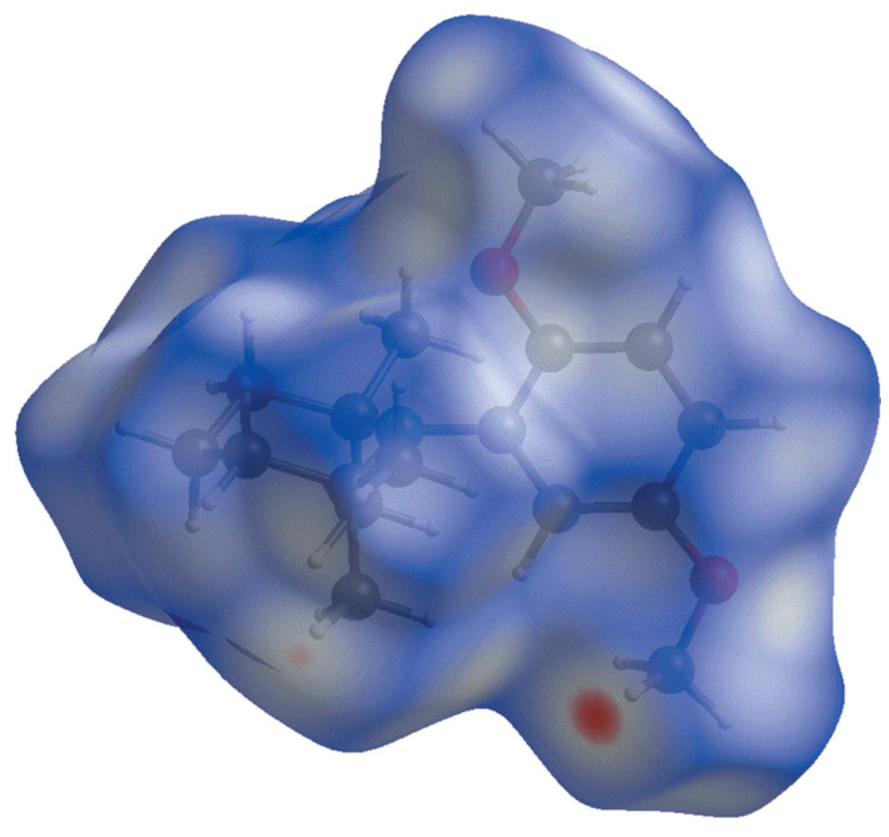

Figure 4

The Hirshfeld surface of compound 3 mapped over $d_{\text {norm }}$, in the colour range -0.1434 to 1.2136 a.u..

$E_{\text {dispersion }}$ and $E_{\text {total }}$ energy components, respectively. The radius of the cylinder is proportional to the magnitude of the interaction energy.

A view of the Hirshfeld surface of $\mathbf{3}$ mapped over $d_{\text {norm }}$ is shown in Fig. 4. The short interatomic $\mathrm{O} \cdots \mathrm{H} / \mathrm{H} \cdots \mathrm{O}$ contacts are indicated by the faint red spots. A full list of short interatomic contacts in the crystal of $\mathbf{3}$ are given in Table 2. The most significant contacts, apart from $\mathrm{H} \cdots \mathrm{H}$ contacts, are (a)

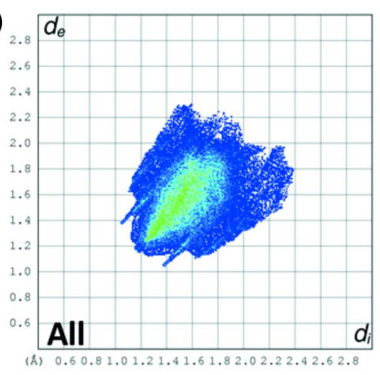

(c)

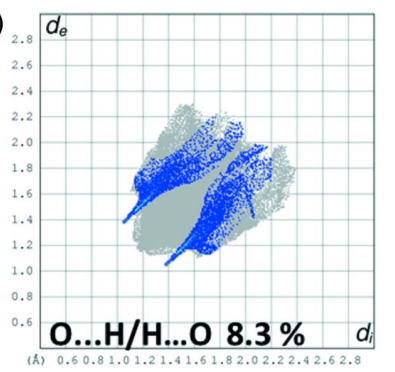

(b)

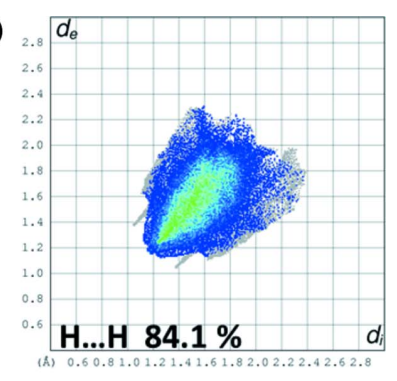

(d)

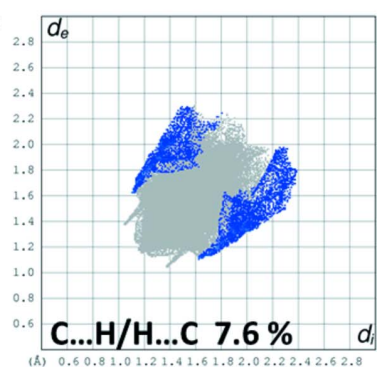

Figure 5

(a) The full two-dimensional fingerprint plot for compound $\mathbf{3}$, and fingerprint plots delineated into $(b) \mathrm{H} \cdots \mathrm{H}$ at $84.1 \%,(c) \mathrm{O} \cdots \mathrm{H} / \mathrm{H} \cdots \mathrm{O}$ at $8.3 \%$ and $(d) \mathrm{C} \cdots \mathrm{H} / \mathrm{H} \cdots \mathrm{C}$ at $7.6 \%$ contacts.
Table 3

Experimental details.
Crystal data

Chemical formula

$M_{\text {r }}$

Crystal system, space group

Temperature (K)

$a, b, c(\AA)$

$\beta\left({ }^{\circ}\right)$

$V\left(\AA^{3}\right)$

Z

Radiation type

$\mu\left(\mathrm{mm}^{-1}\right)$

Crystal size (mm)

Data collection

Diffractometer

No. of measured, independent and observed $[I>2 \sigma(I)]$ reflections

$R_{\text {int }}$

$(\sin \theta / \lambda)_{\max }\left(\AA^{-1}\right)$

Refinement

$R\left[F^{2}>2 \sigma\left(F^{2}\right)\right], w R\left(F^{2}\right), S$

No. of reflections

No. of parameters

$\mathrm{H}$-atom treatment

$\Delta \rho_{\max }, \Delta \rho_{\min }\left(\mathrm{e} \AA^{-3}\right)$
$\mathrm{C}_{17} \mathrm{H}_{27} \mathrm{NO}_{2}$

277.39

Monoclinic, $P 2_{1} / c$

173

6.8817 (10), 28.249 (4), 8.1369 (13)

99.649 (12)

1559.4 (4)

4

Mo $K \alpha$

0.08

$0.40 \times 0.10 \times 0.10$

10596, 2770, 1695

0.085

0.599

$0.037,0.073,0.83$

2770

188

$\mathrm{H}$-atom parameters constrained $0.13,-0.13$
STOE IPDS 2

Computer programs: $X$-AREA and X-RED32 (Stoe \& Cie, 2005), SHELXS97 (Sheldrick, 2008), SHELXL2018/3 (Sheldrick, 2015), PLATON (Spek, 2020), Mercury (Macrae et al., 2020) and publCIF (Westrip, 2010).

$\mathrm{O} \cdot \mathrm{H}$ and $\mathrm{C} \cdot \mathrm{H}$ contacts as confirmed by the two-dimensional fingerprint plots (Fig. 5). The principal intermolecular contacts for 3, are delineated into $\mathrm{H} \cdots \mathrm{H}$ at $84.1 \%$ (Fig. $5 b$ ), $\mathrm{O} \cdots \mathrm{H} / \mathrm{H} \cdots \mathrm{O}$ at $8.3 \%$ (Fig. $5 c$ ) and $\mathrm{C} \cdot \mathrm{H} / \mathrm{H} \cdots \mathrm{C}$ at $7.6 \%$ (Fig. $5 d$ ) contacts. The intermolecular contacts are therefore dominated by dispersion forces ( $\mathrm{H} \cdots \mathrm{H}$ at $84.1 \%$; Fig. $5 b)$. This is confirmed by the energy frameworks shown in Fig. 6. The energy frameworks were adjusted to the same scale factor of 80 with a cut-off value of $5 \mathrm{~kJ} \mathrm{~mol}^{-1}$ within $2 \times 2 \times 2$ unit cells, and obtained using the wave function calculated at the $\mathrm{HF} / 3-21 \mathrm{G}$ level of theory.

\section{Database survey}

A search of the Cambridge Structural Database (CSD, Version 5.41, last update March 2020; Groom et al., 2016) for 1-(phenyl)-2,2,6,6-tetramethylpiperidines gave 26 hits (see file $\mathrm{S} 1$ in the supporting information). A number of these structures involve heteroaryl and heterocyclic aluminium compounds, see for example CSD refcodes CEGLUY, CEGMF, CEGMEJ, CEGMIN, CEGMOT and CEGMUZ (Chen et al., 2017). They also include a number of borohydride derivatives, see for example CSD refcodes JAKZON, JAKZUT, JALBAC and JALBEG (Chernichenko et al., 2017). Only one compound has a methoxy substituent, viz. 1-(2-iodo3-methoxyphenyl)-2,2,6,6-tetramethylpiperidine (VAPCUM; Crosbie et al., 2012). In these eleven compounds, the piperidine ring has a chair conformation with the mean plane of the four planar $\mathrm{C}$ atoms being inclined to the plane of the benzene ring by dihedral angles varying from $c a 83.0$ to $90.0^{\circ}$. In compound 3 this dihedral angle is similar at $88.34(9)^{\circ}$. 

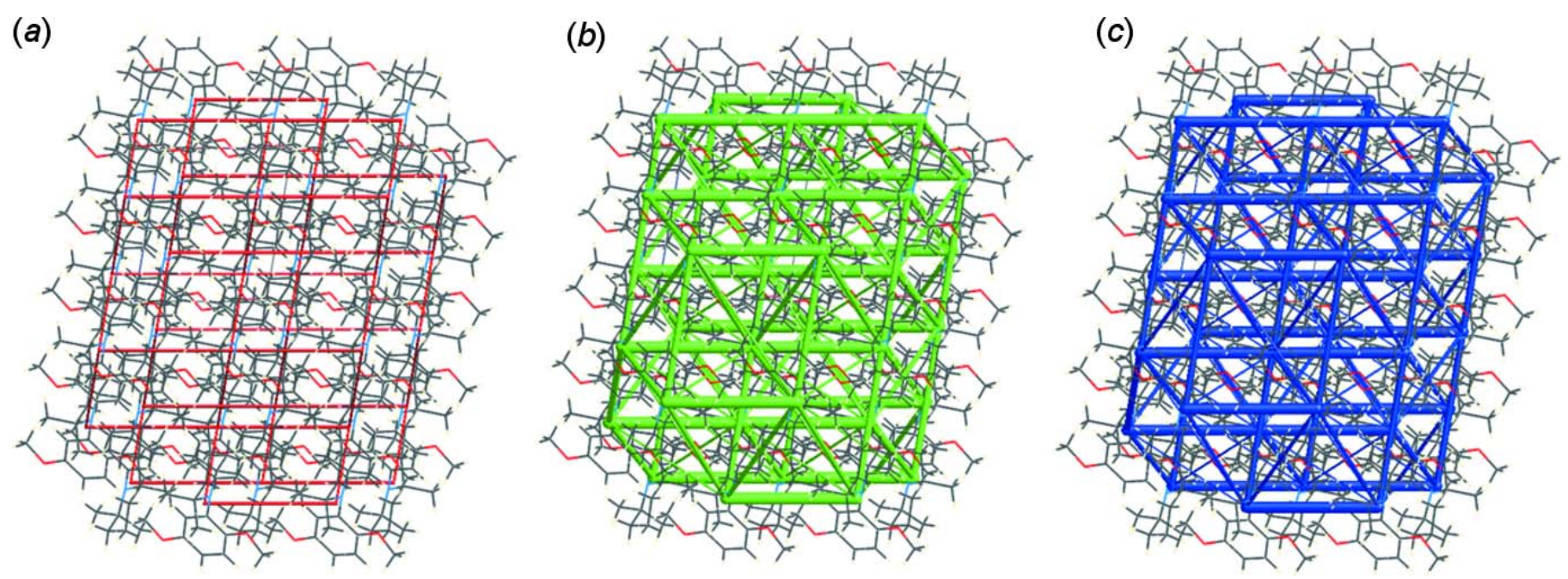

Figure 6

The energy frameworks viewed down the $b$-axis direction comprising $(a)$ electrostatic potential forces, $(b)$ dispersion forces and $(c)$ total energy for a cluster about a reference molecule of 3 . The energy frameworks were adjusted to the same scale factor of 80 with a cut-off value of $5 \mathrm{~kJ} \mathrm{~mol}^{-1}$ within $2 \times$ $2 \times 2$ unit cells.

\section{Synthesis and crystallization}

The synthesis of compound $\mathbf{3}$ is illustrated in Fig. 1. It arises as a result of the condensation of 2-bromo-1,4-dimethoxybenzene (1) with tetramethylpiperidene (HTMP). It is a side product obtained during the synthesis of methyl 3,6-dimethoxy-2-(2-methoxy-2-oxoethyl)benzoate (2) (Tiouabi, 2005). Colourless rod-like crystals of $\mathbf{3}$ were obtained by slow evaporation at room temperature of a solution in acetone.There are no analytical or spectroscopic data available for compound $\mathbf{3}$.

\section{Refinement details}

Crystal data, data collection and structure refinement details are summarized in Table 3. The hydrogen atoms were fixed geometrically $(\mathrm{C}-\mathrm{H}=0.95-0.99 \AA)$ and allowed to ride on their parent atoms with $U_{\text {iso }}(\mathrm{H})=1.5 U_{\text {eq }}(\mathrm{C}$-methyl $)$ and $1.2 U_{\mathrm{eq}}(\mathrm{C})$ for other $\mathrm{H}$ atoms.

\section{Acknowledgements}

RT and HSE are grateful to the University of Neuchâtel for their support over the years.

\section{Funding information}

Funding for this research was provided by: Swiss National Science Foundation and the University of Neuchâtel.

\section{References}

Chen, S., Li, B., Wang, X., Huang, Y., Li, J., Zhu, H., Zhao, L., Frenking, G. \& Roesky, H. W. (2017). Chem. Eur. J. 23, 1363313637.

Chernichenko, K., Kótai, B., Nieger, M., Heikkinen, S., Pápai, I. \& Repo, T. (2017). Dalton Trans. 46, 2263-2269.

Crosbie, E., Kennedy, A. R., Mulvey, R. E. \& Robertson, S. D. (2012). Dalton Trans. 41, 1832-1839.

Groom, C. R., Bruno, I. J., Lightfoot, M. P. \& Ward, S. C. (2016). Acta Cryst. B72, 171-179.

Macrae, C. F., Sovago, I., Cottrell, S. J., Galek, P. T. A., McCabe, P., Pidcock, E., Platings, M., Shields, G. P., Stevens, J. S., Towler, M. \& Wood, P. A. (2020). J. Appl. Cryst. 53, 226-235.

McKinnon, J. J., Jayatilaka, D. \& Spackman, M. A. (2007). Chem. Commun. pp. 3814-3816.

Sheldrick, G. M. (2008). Acta Cryst. A64, 112-122.

Sheldrick, G. M. (2015). Acta Cryst. C71, 3-8.

Spackman, M. A. \& Jayatilaka, D. (2009). CrystEngComm, 11, 19-32.

Spek, A. L. (2020). Acta Cryst. E76, 1-11.

Stoe \& Cie. (2005). $X$-AREA and $X$-RED32. Stoe \& Cie GmbH, Darmstadt, Germany.

Tan, S. L., Jotani, M. M. \& Tiekink, E. R. T. (2019). Acta Cryst. E75, 308-318.

Tiouabi, M. (2005). PhD Thesis. University of Neuchâtel, Switzerland.

Turner, M. J., McKinnon, J. J., Wolff, S. K., Grimwood, D. J., Spackman, P. R., Jayatilaka, D. \& Spackman, M. A. (2017). CrystalExplorer17. University of Western Australia. http://hirshfeldsurface.net

Turner, M. J., Thomas, S. P., Shi, M. W., Jayatilaka, D. \& Spackman, M. A. (2015). Chem. Commun. 51, 3735-3738.

Westrip, S. P. (2010). J. Appl. Cryst. 43, 920-925. 


\section{supporting information}

Acta Cryst. (2020). E76, 794-797 [https://doi.org/10.1107/S2056989020005952]

\section{The crystal structure and Hirshfeld surface analysis of 1-(2,5-dimethoxy- phenyl)-2,2,6,6-tetramethylpiperidine}

\section{Mustapha Tiouabi, Raphaël Tabacchi and Helen Stoeckli-Evans}

Computing details

Data collection: $X$-AREA (Stoe \& Cie, 2005); cell refinement: $X$-AREA (Stoe \& Cie, 2005); data reduction: $X$-RED32

(Stoe \& Cie, 2005); program(s) used to solve structure: SHELXS97 (Sheldrick, 2008); program(s) used to refine structure: SHELXL2018/3 (Sheldrick, 2015); molecular graphics: PLATON (Spek, 2020) and Mercury (Macrae et al., 2020); software used to prepare material for publication: SHELXL2018/3 (Sheldrick, 2015), PLATON (Spek, 2020) and publCIF (Westrip, 2010).

1-(2,5-Dimethoxyphenyl)-2,2,6,6-tetramethylpiperidine

Crystal data

$\mathrm{C}_{17} \mathrm{H}_{27} \mathrm{NO}_{2}$

$M_{r}=277.39$

Monoclinic, $P 2_{1} / c$

$a=6.8817(10) \AA$

$b=28.249(4) \AA$

$c=8.1369(13) \AA$

$\beta=99.649(12)^{\circ}$

$V=1559.4(4) \AA^{3}$

$Z=4$

Data collection

STOE IPDS 2

diffractometer

Radiation source: fine-focus sealed tube

Plane graphite monochromator

$\varphi+\omega$ scans

10596 measured reflections

2770 independent reflections

\section{Refinement}

Refinement on $F^{2}$

Least-squares matrix: full

$R\left[F^{2}>2 \sigma\left(F^{2}\right)\right]=0.037$

$w R\left(F^{2}\right)=0.073$

$S=0.83$

2770 reflections

188 parameters

0 restraints

Primary atom site location: structure-invariant direct methods
$F(000)=608$

$D_{\mathrm{x}}=1.182 \mathrm{Mg} \mathrm{m}^{-3}$

Mo $K \alpha$ radiation, $\lambda=0.71073 \AA$

Cell parameters from 4940 reflections

$\theta=1.4-25.5^{\circ}$

$\mu=0.08 \mathrm{~mm}^{-1}$

$T=173 \mathrm{~K}$

Rod, colourless

$0.40 \times 0.10 \times 0.10 \mathrm{~mm}$

1695 reflections with $I>2 \sigma(I)$

$R_{\text {int }}=0.085$

$\theta_{\max }=25.2^{\circ}, \theta_{\min }=1.4^{\circ}$

$h=-8 \rightarrow 8$

$k=-33 \rightarrow 33$

$l=-9 \rightarrow 9$

Secondary atom site location: difference Fourier map

Hydrogen site location: inferred from neighbouring sites

$\mathrm{H}$-atom parameters constrained

$w=1 /\left[\sigma^{2}\left(F_{0}^{2}\right)+(0.0237 P)^{2}\right]$

where $P=\left(F_{\mathrm{o}}^{2}+2 F_{\mathrm{c}}^{2}\right) / 3$

$(\Delta / \sigma)_{\max }<0.001$

$\Delta \rho_{\max }=0.13 \mathrm{e} \AA^{-3}$

$\Delta \rho_{\min }=-0.13 \mathrm{e} \AA^{-3}$ 
Extinction correction: (SHELXL2018/3; Sheldrick, 2015),

$\mathrm{Fc}^{*}=\mathrm{kFc}\left[1+0.001 \mathrm{xFc}^{2} \lambda^{3} / \sin (2 \theta)\right]^{-1 / 4}$

Extinction coefficient: $0.0127(13)$

\section{Special details}

Geometry. All esds (except the esd in the dihedral angle between two 1.s. planes) are estimated using the full covariance matrix. The cell esds are taken into account individually in the estimation of esds in distances, angles and torsion angles; correlations between esds in cell parameters are only used when they are defined by crystal symmetry. An approximate (isotropic) treatment of cell esds is used for estimating esds involving l.s. planes.

Fractional atomic coordinates and isotropic or equivalent isotropic displacement parameters $\left(\AA^{2}\right)$

\begin{tabular}{|c|c|c|c|c|}
\hline & $x$ & $y$ & $z$ & $U_{\text {iso }} * / U_{\text {eq }}$ \\
\hline $\mathrm{O} 1$ & $-0.15231(15)$ & $0.11464(4)$ & $0.48898(14)$ & $0.0359(3)$ \\
\hline $\mathrm{O} 2$ & $0.47503(17)$ & $0.23289(4)$ & $0.44354(15)$ & $0.0389(3)$ \\
\hline N1 & $0.17336(17)$ & $0.10517(4)$ & $0.72612(15)$ & $0.0239(3)$ \\
\hline $\mathrm{C} 1$ & $0.1701(2)$ & $0.14010(5)$ & $0.59863(18)$ & $0.0240(4)$ \\
\hline $\mathrm{C} 2$ & $0.0023(2)$ & $0.14458(5)$ & 0.4777 (2) & $0.0274(4)$ \\
\hline $\mathrm{C} 3$ & $-0.0050(2)$ & $0.17837(6)$ & $0.3529(2)$ & $0.0320(4)$ \\
\hline $\mathrm{H} 3$ & -0.120713 & 0.181465 & 0.271592 & $0.038^{*}$ \\
\hline $\mathrm{C} 4$ & $0.1545(2)$ & $0.20752(6)$ & $0.3461(2)$ & $0.0326(4)$ \\
\hline $\mathrm{H} 4$ & 0.148437 & 0.230424 & 0.259885 & $0.039 *$ \\
\hline $\mathrm{C} 5$ & $0.3219(2)$ & $0.20347(5)$ & $0.4637(2)$ & $0.0286(4)$ \\
\hline C6 & $0.3285(2)$ & $0.17020(5)$ & $0.58934(19)$ & $0.0264(4)$ \\
\hline H6 & 0.443892 & 0.167808 & 0.671343 & $0.032 *$ \\
\hline $\mathrm{C} 7$ & $-0.3053(2)$ & $0.11190(7)$ & $0.3485(2)$ & $0.0420(5)$ \\
\hline $\mathrm{H} 7 \mathrm{C}$ & -0.398183 & 0.086893 & 0.366432 & $0.063^{*}$ \\
\hline H7B & -0.248449 & 0.104630 & 0.248721 & $0.063^{*}$ \\
\hline $\mathrm{H} 7 \mathrm{~A}$ & -0.374711 & 0.142279 & 0.333365 & $0.063^{*}$ \\
\hline $\mathrm{C} 8$ & $0.6384(3)$ & $0.23518(6)$ & $0.5748(2)$ & $0.0407(5)$ \\
\hline H8A & 0.703032 & 0.204187 & 0.588607 & $0.061 *$ \\
\hline H8B & 0.592985 & 0.244041 & 0.678454 & $0.061^{*}$ \\
\hline $\mathrm{H} 8 \mathrm{C}$ & 0.732223 & 0.258909 & 0.548061 & $0.061 *$ \\
\hline C9 & $0.1554(2)$ & $0.12313(5)$ & 0.89467 (19) & $0.0269(4)$ \\
\hline $\mathrm{C} 10$ & $0.0977(2)$ & $0.08181(6)$ & $0.9977(2)$ & $0.0342(4)$ \\
\hline H10A & 0.103522 & 0.092378 & 1.114443 & $0.041 *$ \\
\hline H10B & -0.040201 & 0.072692 & 0.954118 & $0.041 *$ \\
\hline C11 & $0.2287(2)$ & $0.03873(6)$ & 0.9958 (2) & $0.0381(5)$ \\
\hline H11A & 0.365874 & 0.046683 & 1.046272 & $0.046^{*}$ \\
\hline H11B & 0.182345 & 0.012857 & 1.061593 & $0.046^{*}$ \\
\hline $\mathrm{C} 12$ & $0.2215(3)$ & $0.02299(6)$ & $0.8172(2)$ & $0.0364(4)$ \\
\hline H12B & 0.084985 & 0.013326 & 0.770843 & $0.044 *$ \\
\hline $\mathrm{H} 12 \mathrm{~A}$ & 0.307259 & -0.005084 & 0.815714 & $0.044^{*}$ \\
\hline $\mathrm{C} 13$ & $0.2870(2)$ & $0.06129(5)$ & $0.7049(2)$ & $0.0294(4)$ \\
\hline C14 & $-0.0136(2)$ & $0.15863(6)$ & $0.8748(2)$ & $0.0352(4)$ \\
\hline $\mathrm{H} 14 \mathrm{C}$ & -0.039727 & 0.167933 & 0.985055 & $0.053 *$ \\
\hline H14B & -0.132032 & 0.144135 & 0.810935 & $0.053 *$ \\
\hline H14A & 0.022371 & 0.186676 & 0.815626 & $0.053^{*}$ \\
\hline
\end{tabular}




\begin{tabular}{lllll} 
C15 & $0.3403(2)$ & $0.14765(6)$ & $0.9888(2)$ & $0.0361(4)$ \\
H15C & 0.316036 & 0.157724 & 1.098873 & $0.054^{*}$ \\
H15B & 0.371277 & 0.175361 & 0.925566 & $0.054^{*}$ \\
H15A & 0.451420 & 0.125506 & 1.002183 & $0.054^{*}$ \\
C16 & $0.5112(2)$ & $0.06636(6)$ & $0.7429(2)$ & $0.0350(4)$ \\
H16C & 0.572685 & 0.036915 & 0.713805 & $0.053^{*}$ \\
H16B & 0.552574 & 0.072901 & 0.861923 & $0.053^{*}$ \\
H16A & 0.552219 & 0.092519 & 0.677366 & $0.053^{*}$ \\
C17 & $0.2301(2)$ & $0.04478(6)$ & $0.5248(2)$ & $0.0387(5)$ \\
H17A & 0.280543 & 0.067303 & 0.450547 & $0.058^{*}$ \\
H17B & 0.086213 & 0.042978 & 0.495777 & $0.058^{*}$ \\
H17C & 0.287008 & 0.013449 & 0.512256 & $0.058^{*}$ \\
\hline
\end{tabular}

Atomic displacement parameters $\left(\AA^{2}\right)$

\begin{tabular}{lllllll}
\hline & $U^{11}$ & $U^{22}$ & $U^{33}$ & $U^{12}$ & $U^{13}$ & $U^{23}$ \\
\hline O1 & $0.0285(6)$ & $0.0478(7)$ & $0.0295(7)$ & $-0.0066(5)$ & $-0.0007(5)$ & $0.0043(6)$ \\
O2 & $0.0453(7)$ & $0.0328(7)$ & $0.0391(7)$ & $-0.0084(6)$ & $0.0085(6)$ & $0.0091(6)$ \\
N1 & $0.0296(7)$ & $0.0217(7)$ & $0.0205(7)$ & $0.0021(6)$ & $0.0045(6)$ & $0.0027(6)$ \\
C1 & $0.0292(9)$ & $0.0225(8)$ & $0.0210(9)$ & $0.0038(7)$ & $0.0058(7)$ & $-0.0006(7)$ \\
C2 & $0.0276(9)$ & $0.0319(9)$ & $0.0233(9)$ & $0.0011(7)$ & $0.0063(8)$ & $-0.0023(7)$ \\
C3 & $0.0353(10)$ & $0.0384(10)$ & $0.0218(9)$ & $0.0095(8)$ & $0.0032(8)$ & $0.0026(8)$ \\
C4 & $0.0465(11)$ & $0.0275(9)$ & $0.0256(9)$ & $0.0094(8)$ & $0.0111(9)$ & $0.0059(7)$ \\
C5 & $0.0362(9)$ & $0.0229(8)$ & $0.0283(9)$ & $0.0011(7)$ & $0.0098(8)$ & $0.0005(7)$ \\
C6 & $0.0304(9)$ & $0.0230(8)$ & $0.0251(8)$ & $0.0027(7)$ & $0.0024(7)$ & $-0.0004(7)$ \\
C7 & $0.0308(10)$ & $0.0594(12)$ & $0.0329(10)$ & $-0.0019(9)$ & $-0.0032(8)$ & $-0.0076(9)$ \\
C8 & $0.0476(11)$ & $0.0392(11)$ & $0.0374(11)$ & $-0.0115(9)$ & $0.0136(10)$ & $-0.0044(8)$ \\
C9 & $0.0334(9)$ & $0.0276(9)$ & $0.0197(8)$ & $0.0020(7)$ & $0.0045(7)$ & $0.0017(7)$ \\
C10 & $0.0368(10)$ & $0.0400(10)$ & $0.0266(9)$ & $0.0011(8)$ & $0.0082(8)$ & $0.0080(8)$ \\
C11 & $0.0397(10)$ & $0.0337(10)$ & $0.0409(11)$ & $0.0013(8)$ & $0.0068(9)$ & $0.0146(8)$ \\
C12 & $0.0379(10)$ & $0.0248(9)$ & $0.0472(11)$ & $0.0019(8)$ & $0.0088(9)$ & $0.0065(8)$ \\
C13 & $0.0318(9)$ & $0.0225(8)$ & $0.0340(10)$ & $0.0007(7)$ & $0.0063(8)$ & $0.0014(7)$ \\
C14 & $0.0428(10)$ & $0.0355(10)$ & $0.0284(10)$ & $0.0095(8)$ & $0.0093(8)$ & $0.0006(8)$ \\
C15 & $0.0427(10)$ & $0.0369(10)$ & $0.0270(9)$ & $-0.0030(8)$ & $0.0010(8)$ & $-0.0032(8)$ \\
C16 & $0.0343(9)$ & $0.0309(9)$ & $0.0403(10)$ & $0.0064(8)$ & $0.0076(8)$ & $0.0036(8)$ \\
C17 & $0.0426(11)$ & $0.0301(9)$ & $0.0437(11)$ & $0.0007(8)$ & $0.0085(9)$ & $-0.0079(9)$ \\
& & & & & & \\
\hline
\end{tabular}

Geometric parameters $\left(\AA,{ }^{\circ}\right)$

\begin{tabular}{llll}
\hline $\mathrm{O} 1-\mathrm{C} 2$ & $1.3743(19)$ & $\mathrm{C} 9-\mathrm{C} 15$ & $1.536(2)$ \\
$\mathrm{O} 1-\mathrm{C} 7$ & $1.4204(18)$ & $\mathrm{C} 10-\mathrm{C} 11$ & $1.516(2)$ \\
$\mathrm{O} 2-\mathrm{C} 5$ & $1.3732(19)$ & $\mathrm{C} 10-\mathrm{H} 10 \mathrm{~A}$ & 0.9900 \\
$\mathrm{O} 2-\mathrm{C} 8$ & $1.416(2)$ & $\mathrm{C} 10-\mathrm{H} 10 \mathrm{~B}$ & 0.9900 \\
$\mathrm{~N} 1-\mathrm{C} 1$ & $1.4293(19)$ & $\mathrm{C} 11-\mathrm{C} 12$ & $1.513(2)$ \\
$\mathrm{N} 1-\mathrm{C} 9$ & $1.4867(19)$ & $\mathrm{C} 11-\mathrm{H} 11 \mathrm{~A}$ & 0.9900 \\
$\mathrm{~N} 1-\mathrm{C} 13$ & $1.4908(19)$ & $\mathrm{C} 11-\mathrm{H} 11 \mathrm{~B}$ & 0.9900 \\
$\mathrm{C} 1-\mathrm{C} 2$ & $1.392(2)$ & $\mathrm{C} 12-\mathrm{C} 13$ & $1.532(2)$ \\
$\mathrm{C} 1-\mathrm{C} 6$ & $1.394(2)$ & $\mathrm{C} 12-\mathrm{H} 12 \mathrm{~B}$ & 0.9900
\end{tabular}




\begin{tabular}{|c|c|c|c|}
\hline $\mathrm{C} 2-\mathrm{C} 3$ & $1.388(2)$ & $\mathrm{C} 12-\mathrm{H} 12 \mathrm{~A}$ & 0.9900 \\
\hline $\mathrm{C} 3-\mathrm{C} 4$ & $1.381(2)$ & $\mathrm{C} 13-\mathrm{C} 17$ & $1.526(2)$ \\
\hline $\mathrm{C} 3-\mathrm{H} 3$ & 0.9500 & $\mathrm{C} 13-\mathrm{C} 16$ & $1.529(2)$ \\
\hline $\mathrm{C} 4-\mathrm{C} 5$ & $1.374(2)$ & $\mathrm{C} 14-\mathrm{H} 14 \mathrm{C}$ & 0.9800 \\
\hline $\mathrm{C} 4-\mathrm{H} 4$ & 0.9500 & C14-H14B & 0.9800 \\
\hline $\mathrm{C} 5-\mathrm{C} 6$ & $1.384(2)$ & $\mathrm{C} 14-\mathrm{H} 14 \mathrm{~A}$ & 0.9800 \\
\hline C6-H6 & 0.9500 & $\mathrm{C} 15-\mathrm{H} 15 \mathrm{C}$ & 0.9800 \\
\hline $\mathrm{C} 7-\mathrm{H} 7 \mathrm{C}$ & 0.9800 & C15-H15B & 0.9800 \\
\hline C7-H7B & 0.9800 & C15-H15A & 0.9800 \\
\hline $\mathrm{C} 7-\mathrm{H} 7 \mathrm{~A}$ & 0.9800 & $\mathrm{C} 16-\mathrm{H} 16 \mathrm{C}$ & 0.9800 \\
\hline $\mathrm{C} 8-\mathrm{H} 8 \mathrm{~A}$ & 0.9800 & C16-H16B & 0.9800 \\
\hline $\mathrm{C} 8-\mathrm{H} 8 \mathrm{~B}$ & 0.9800 & $\mathrm{C} 16-\mathrm{H} 16 \mathrm{~A}$ & 0.9800 \\
\hline $\mathrm{C} 8-\mathrm{H} 8 \mathrm{C}$ & 0.9800 & C17-H17A & 0.9800 \\
\hline $\mathrm{C} 9-\mathrm{C} 14$ & $1.524(2)$ & C17-H17B & 0.9800 \\
\hline $\mathrm{C} 9-\mathrm{C} 10$ & $1.528(2)$ & $\mathrm{C} 17-\mathrm{H} 17 \mathrm{C}$ & 0.9800 \\
\hline $\mathrm{C} 2-\mathrm{O} 1-\mathrm{C} 7$ & $117.22(13)$ & $\mathrm{C} 9-\mathrm{C} 10-\mathrm{H} 10 \mathrm{~B}$ & 108.9 \\
\hline $\mathrm{C} 5-\mathrm{O} 2-\mathrm{C} 8$ & $117.75(13)$ & $\mathrm{H} 10 \mathrm{~A}-\mathrm{C} 10-\mathrm{H} 10 \mathrm{~B}$ & 107.7 \\
\hline $\mathrm{C} 1-\mathrm{N} 1-\mathrm{C} 9$ & $116.13(11)$ & $\mathrm{C} 12-\mathrm{C} 11-\mathrm{C} 10$ & $108.78(14)$ \\
\hline $\mathrm{C} 1-\mathrm{N} 1-\mathrm{C} 13$ & $115.76(12)$ & $\mathrm{C} 12-\mathrm{C} 11-\mathrm{H} 11 \mathrm{~A}$ & 109.9 \\
\hline $\mathrm{C} 9-\mathrm{N} 1-\mathrm{C} 13$ & $121.11(11)$ & $\mathrm{C} 10-\mathrm{C} 11-\mathrm{H} 11 \mathrm{~A}$ & 109.9 \\
\hline $\mathrm{C} 2-\mathrm{C} 1-\mathrm{C} 6$ & $118.02(14)$ & $\mathrm{C} 12-\mathrm{C} 11-\mathrm{H} 11 \mathrm{~B}$ & 109.9 \\
\hline $\mathrm{C} 2-\mathrm{C} 1-\mathrm{N} 1$ & $119.07(14)$ & $\mathrm{C} 10-\mathrm{C} 11-\mathrm{H} 11 \mathrm{~B}$ & 109.9 \\
\hline $\mathrm{C} 6-\mathrm{C} 1-\mathrm{N} 1$ & $122.90(13)$ & $\mathrm{H} 11 \mathrm{~A}-\mathrm{C} 11-\mathrm{H} 11 \mathrm{~B}$ & 108.3 \\
\hline $\mathrm{O} 1-\mathrm{C} 2-\mathrm{C} 3$ & $122.57(14)$ & $\mathrm{C} 11-\mathrm{C} 12-\mathrm{C} 13$ & $113.56(14)$ \\
\hline $\mathrm{O} 1-\mathrm{C} 2-\mathrm{C} 1$ & $117.18(14)$ & $\mathrm{C} 11-\mathrm{C} 12-\mathrm{H} 12 \mathrm{~B}$ & 108.9 \\
\hline $\mathrm{C} 3-\mathrm{C} 2-\mathrm{C} 1$ & $120.24(15)$ & $\mathrm{C} 13-\mathrm{C} 12-\mathrm{H} 12 \mathrm{~B}$ & 108.9 \\
\hline $\mathrm{C} 4-\mathrm{C} 3-\mathrm{C} 2$ & $120.51(15)$ & $\mathrm{C} 11-\mathrm{C} 12-\mathrm{H} 12 \mathrm{~A}$ & 108.9 \\
\hline $\mathrm{C} 4-\mathrm{C} 3-\mathrm{H} 3$ & 119.7 & $\mathrm{C} 13-\mathrm{C} 12-\mathrm{H} 12 \mathrm{~A}$ & 108.9 \\
\hline $\mathrm{C} 2-\mathrm{C} 3-\mathrm{H} 3$ & 119.7 & $\mathrm{H} 12 \mathrm{~B}-\mathrm{C} 12-\mathrm{H} 12 \mathrm{~A}$ & 107.7 \\
\hline $\mathrm{C} 5-\mathrm{C} 4-\mathrm{C} 3$ & $120.13(15)$ & $\mathrm{N} 1-\mathrm{C} 13-\mathrm{C} 17$ & $108.08(12)$ \\
\hline $\mathrm{C} 5-\mathrm{C} 4-\mathrm{H} 4$ & 119.9 & $\mathrm{~N} 1-\mathrm{C} 13-\mathrm{C} 16$ & $115.46(12)$ \\
\hline $\mathrm{C} 3-\mathrm{C} 4-\mathrm{H} 4$ & 119.9 & $\mathrm{C} 17-\mathrm{C} 13-\mathrm{C} 16$ & $108.10(14)$ \\
\hline $\mathrm{O} 2-\mathrm{C} 5-\mathrm{C} 4$ & $115.91(14)$ & $\mathrm{N} 1-\mathrm{C} 13-\mathrm{C} 12$ & $107.81(13)$ \\
\hline $\mathrm{O} 2-\mathrm{C} 5-\mathrm{C} 6$ & $124.65(14)$ & $\mathrm{C} 17-\mathrm{C} 13-\mathrm{C} 12$ & $107.65(13)$ \\
\hline $\mathrm{C} 4-\mathrm{C} 5-\mathrm{C} 6$ & $119.42(15)$ & $\mathrm{C} 16-\mathrm{C} 13-\mathrm{C} 12$ & $109.49(13)$ \\
\hline $\mathrm{C} 5-\mathrm{C} 6-\mathrm{C} 1$ & $121.67(14)$ & $\mathrm{C} 9-\mathrm{C} 14-\mathrm{H} 14 \mathrm{C}$ & 109.5 \\
\hline $\mathrm{C} 5-\mathrm{C} 6-\mathrm{H} 6$ & 119.2 & C9- $14-\mathrm{H} 14 \mathrm{~B}$ & 109.5 \\
\hline $\mathrm{C} 1-\mathrm{C} 6-\mathrm{H} 6$ & 119.2 & $\mathrm{H} 14 \mathrm{C}-\mathrm{C} 14-\mathrm{H} 14 \mathrm{~B}$ & 109.5 \\
\hline $\mathrm{O} 1-\mathrm{C} 7-\mathrm{H} 7 \mathrm{C}$ & 109.5 & $\mathrm{C} 9-\mathrm{C} 14-\mathrm{H} 14 \mathrm{~A}$ & 109.5 \\
\hline $\mathrm{O} 1-\mathrm{C} 7-\mathrm{H} 7 \mathrm{~B}$ & 109.5 & $\mathrm{H} 14 \mathrm{C}-\mathrm{C} 14-\mathrm{H} 14 \mathrm{~A}$ & 109.5 \\
\hline $\mathrm{H} 7 \mathrm{C}-\mathrm{C} 7-\mathrm{H} 7 \mathrm{~B}$ & 109.5 & $\mathrm{H} 14 \mathrm{~B}-\mathrm{C} 14-\mathrm{H} 14 \mathrm{~A}$ & 109.5 \\
\hline $\mathrm{O} 1-\mathrm{C} 7-\mathrm{H} 7 \mathrm{~A}$ & 109.5 & $\mathrm{C} 9-\mathrm{C} 15-\mathrm{H} 15 \mathrm{C}$ & 109.5 \\
\hline $\mathrm{H} 7 \mathrm{C}-\mathrm{C} 7-\mathrm{H} 7 \mathrm{~A}$ & 109.5 & C9-C15-H15B & 109.5 \\
\hline $\mathrm{H} 7 \mathrm{~B}-\mathrm{C} 7-\mathrm{H} 7 \mathrm{~A}$ & 109.5 & $\mathrm{H} 15 \mathrm{C}-\mathrm{C} 15-\mathrm{H} 15 \mathrm{~B}$ & 109.5 \\
\hline $\mathrm{O} 2-\mathrm{C} 8-\mathrm{H} 8 \mathrm{~A}$ & 109.5 & C9- $115-\mathrm{H} 15 \mathrm{~A}$ & 109.5 \\
\hline $\mathrm{O} 2-\mathrm{C} 8-\mathrm{H} 8 \mathrm{~B}$ & 109.5 & $\mathrm{H} 15 \mathrm{C}-\mathrm{C} 15-\mathrm{H} 15 \mathrm{~A}$ & 109.5 \\
\hline $\mathrm{H} 8 \mathrm{~A}-\mathrm{C} 8-\mathrm{H} 8 \mathrm{~B}$ & 109.5 & $\mathrm{H} 15 \mathrm{~B}-\mathrm{C} 15-\mathrm{H} 15 \mathrm{~A}$ & 109.5 \\
\hline
\end{tabular}




\begin{tabular}{|c|c|c|c|}
\hline $\mathrm{O} 2-\mathrm{C} 8-\mathrm{H} 8 \mathrm{C}$ & 109.5 & $\mathrm{C} 13-\mathrm{C} 16-\mathrm{H} 16 \mathrm{C}$ & 109.5 \\
\hline $\mathrm{H} 8 \mathrm{~A}-\mathrm{C} 8-\mathrm{H} 8 \mathrm{C}$ & 109.5 & $\mathrm{C} 13-\mathrm{C} 16-\mathrm{H} 16 \mathrm{~B}$ & 109.5 \\
\hline $\mathrm{H} 8 \mathrm{~B}-\mathrm{C} 8-\mathrm{H} 8 \mathrm{C}$ & 109.5 & $\mathrm{H} 16 \mathrm{C}-\mathrm{C} 16-\mathrm{H} 16 \mathrm{~B}$ & 109.5 \\
\hline $\mathrm{N} 1-\mathrm{C} 9-\mathrm{C} 14$ & $107.86(12)$ & $\mathrm{C} 13-\mathrm{C} 16-\mathrm{H} 16 \mathrm{~A}$ & 109.5 \\
\hline $\mathrm{N} 1-\mathrm{C} 9-\mathrm{C} 10$ & $108.36(12)$ & $\mathrm{H} 16 \mathrm{C}-\mathrm{C} 16-\mathrm{H} 16 \mathrm{~A}$ & 109.5 \\
\hline $\mathrm{C} 14-\mathrm{C} 9-\mathrm{C} 10$ & $107.30(14)$ & $\mathrm{H} 16 \mathrm{~B}-\mathrm{C} 16-\mathrm{H} 16 \mathrm{~A}$ & 109.5 \\
\hline $\mathrm{N} 1-\mathrm{C} 9-\mathrm{C} 15$ & $115.10(13)$ & $\mathrm{C} 13-\mathrm{C} 17-\mathrm{H} 17 \mathrm{~A}$ & 109.5 \\
\hline $\mathrm{C} 14-\mathrm{C} 9-\mathrm{C} 15$ & $108.04(13)$ & $\mathrm{C} 13-\mathrm{C} 17-\mathrm{H} 17 \mathrm{~B}$ & 109.5 \\
\hline $\mathrm{C} 10-\mathrm{C} 9-\mathrm{C} 15$ & $109.90(13)$ & $\mathrm{H} 17 \mathrm{~A}-\mathrm{C} 17-\mathrm{H} 17 \mathrm{~B}$ & 109.5 \\
\hline $\mathrm{C} 11-\mathrm{C} 10-\mathrm{C} 9$ & $113.44(14)$ & $\mathrm{C} 13-\mathrm{C} 17-\mathrm{H} 17 \mathrm{C}$ & 109.5 \\
\hline $\mathrm{C} 11-\mathrm{C} 10-\mathrm{H} 10 \mathrm{~A}$ & 108.9 & $\mathrm{H} 17 \mathrm{~A}-\mathrm{C} 17-\mathrm{H} 17 \mathrm{C}$ & 109.5 \\
\hline C9-C10-H10A & 108.9 & $\mathrm{H} 17 \mathrm{~B}-\mathrm{C} 17-\mathrm{H} 17 \mathrm{C}$ & 109.5 \\
\hline $\mathrm{C} 11-\mathrm{C} 10-\mathrm{H} 10 \mathrm{~B}$ & 108.9 & & \\
\hline $\mathrm{C} 9-\mathrm{N} 1-\mathrm{C} 1-\mathrm{C} 2$ & $-106.25(16)$ & $\mathrm{C} 1-\mathrm{N} 1-\mathrm{C} 9-\mathrm{C} 14$ & $46.76(17)$ \\
\hline $\mathrm{C} 13-\mathrm{N} 1-\mathrm{C} 1-\mathrm{C} 2$ & $102.61(16)$ & $\mathrm{C} 13-\mathrm{N} 1-\mathrm{C} 9-\mathrm{C} 14$ & $-163.75(13)$ \\
\hline $\mathrm{C} 9-\mathrm{N} 1-\mathrm{C} 1-\mathrm{C} 6$ & $73.77(19)$ & $\mathrm{C} 1-\mathrm{N} 1-\mathrm{C} 9-\mathrm{C} 10$ & $162.62(12)$ \\
\hline $\mathrm{C} 13-\mathrm{N} 1-\mathrm{C} 1-\mathrm{C} 6$ & $-77.38(18)$ & $\mathrm{C} 13-\mathrm{N} 1-\mathrm{C} 9-\mathrm{C} 10$ & $-47.89(17)$ \\
\hline $\mathrm{C} 7-\mathrm{O} 1-\mathrm{C} 2-\mathrm{C} 3$ & $13.5(2)$ & $\mathrm{C} 1-\mathrm{N} 1-\mathrm{C} 9-\mathrm{C} 15$ & $-73.91(16)$ \\
\hline $\mathrm{C} 7-\mathrm{O} 1-\mathrm{C} 2-\mathrm{C} 1$ & $-166.84(14)$ & $\mathrm{C} 13-\mathrm{N} 1-\mathrm{C} 9-\mathrm{C} 15$ & $75.58(17)$ \\
\hline $\mathrm{C} 6-\mathrm{C} 1-\mathrm{C} 2-\mathrm{O} 1$ & $-179.96(14)$ & $\mathrm{N} 1-\mathrm{C} 9-\mathrm{C} 10-\mathrm{C} 11$ & $50.81(17)$ \\
\hline $\mathrm{N} 1-\mathrm{C} 1-\mathrm{C} 2-\mathrm{O} 1$ & $0.1(2)$ & $\mathrm{C} 14-\mathrm{C} 9-\mathrm{C} 10-\mathrm{C} 11$ & $167.03(14)$ \\
\hline $\mathrm{C} 6-\mathrm{C} 1-\mathrm{C} 2-\mathrm{C} 3$ & $-0.3(2)$ & $\mathrm{C} 15-\mathrm{C} 9-\mathrm{C} 10-\mathrm{C} 11$ & $-75.74(17)$ \\
\hline $\mathrm{N} 1-\mathrm{C} 1-\mathrm{C} 2-\mathrm{C} 3$ & $179.71(14)$ & $\mathrm{C} 9-\mathrm{C} 10-\mathrm{C} 11-\mathrm{C} 12$ & $-57.94(18)$ \\
\hline $\mathrm{O} 1-\mathrm{C} 2-\mathrm{C} 3-\mathrm{C} 4$ & $-179.64(15)$ & $\mathrm{C} 10-\mathrm{C} 11-\mathrm{C} 12-\mathrm{C} 13$ & $58.42(18)$ \\
\hline $\mathrm{C} 1-\mathrm{C} 2-\mathrm{C} 3-\mathrm{C} 4$ & $0.7(2)$ & $\mathrm{C} 1-\mathrm{N} 1-\mathrm{C} 13-\mathrm{C} 17$ & $-46.30(16)$ \\
\hline $\mathrm{C} 2-\mathrm{C} 3-\mathrm{C} 4-\mathrm{C} 5$ & $-0.4(2)$ & $\mathrm{C} 9-\mathrm{N} 1-\mathrm{C} 13-\mathrm{C} 17$ & $164.11(13)$ \\
\hline $\mathrm{C} 8-\mathrm{O} 2-\mathrm{C} 5-\mathrm{C} 4$ & $170.51(15)$ & $\mathrm{C} 1-\mathrm{N} 1-\mathrm{C} 13-\mathrm{C} 16$ & $74.85(17)$ \\
\hline $\mathrm{C} 8-\mathrm{O} 2-\mathrm{C} 5-\mathrm{C} 6$ & $-11.2(2)$ & $\mathrm{C} 9-\mathrm{N} 1-\mathrm{C} 13-\mathrm{C} 16$ & $-74.74(18)$ \\
\hline $\mathrm{C} 3-\mathrm{C} 4-\mathrm{C} 5-\mathrm{O} 2$ & $177.94(15)$ & $\mathrm{C} 1-\mathrm{N} 1-\mathrm{C} 13-\mathrm{C} 12$ & $-162.40(12)$ \\
\hline $\mathrm{C} 3-\mathrm{C} 4-\mathrm{C} 5-\mathrm{C} 6$ & $-0.4(2)$ & $\mathrm{C} 9-\mathrm{N} 1-\mathrm{C} 13-\mathrm{C} 12$ & $48.01(17)$ \\
\hline $\mathrm{O} 2-\mathrm{C} 5-\mathrm{C} 6-\mathrm{C} 1$ & $-177.36(15)$ & $\mathrm{C} 11-\mathrm{C} 12-\mathrm{C} 13-\mathrm{N} 1$ & $-51.42(17)$ \\
\hline $\mathrm{C} 4-\mathrm{C} 5-\mathrm{C} 6-\mathrm{C} 1$ & $0.9(2)$ & $\mathrm{C} 11-\mathrm{C} 12-\mathrm{C} 13-\mathrm{C} 17$ & $-167.79(14)$ \\
\hline $\mathrm{C} 2-\mathrm{C} 1-\mathrm{C} 6-\mathrm{C} 5$ & $-0.5(2)$ & $\mathrm{C} 11-\mathrm{C} 12-\mathrm{C} 13-\mathrm{C} 16$ & $74.93(18)$ \\
\hline $\mathrm{N} 1-\mathrm{C} 1-\mathrm{C} 6-\mathrm{C} 5$ & $179.49(14)$ & & \\
\hline
\end{tabular}

Hydrogen-bond geometry $\left(\AA,{ }^{\circ}\right)$

\begin{tabular}{lllll}
\hline$D-\mathrm{H} \cdots A$ & $D-\mathrm{H}$ & $\mathrm{H} \cdots A$ & $D \cdots A$ & $D-\mathrm{H} \cdots A$ \\
\hline $\mathrm{C} 8-\mathrm{H} 8 B \cdots \mathrm{O} 2^{\mathrm{i}}$ & 0.98 & 2.51 & $3.495(2)$ & 180 \\
\hline
\end{tabular}

Symmetry code: (i) $x,-y+1 / 2, z+1 / 2$. 